\title{
Timetable Design for Urban Rail Line with Capacity Constraints
}

\author{
Yu-Ting Zhu, ${ }^{1,2}$ Bao-Hua Mao, ${ }^{1,2}$ Lu Liu, ${ }^{1}$ and Ming-Gao Li ${ }^{1}$ \\ ${ }^{1}$ MOE Key Laboratory for Urban Transportation Complex Systems Theory and Technology, Beijing Jiaotong University, \\ Beijing 100044, China \\ ${ }^{2}$ School of Traffic and Transportation, Beijing Jiaotong University, Beijing 100044, China
}

Correspondence should be addressed to Bao-Hua Mao; bhmao@china.com

Received 26 December 2014; Accepted 4 March 2015

Academic Editor: Juan R. Torregrosa

Copyright (C) 2015 Yu-Ting Zhu et al. This is an open access article distributed under the Creative Commons Attribution License, which permits unrestricted use, distribution, and reproduction in any medium, provided the original work is properly cited.

To design an efficient and economical timetable for a heavily congested urban rail corridor, a scheduling model is proposed in this paper. The objective of the proposed model is to find the departure time of trains at the start terminal to minimize the system cost, which includes passenger waiting cost and operating cost. To evaluate the performance of the timetable, a simulation model is developed to simulate the detailed movements of passengers and trains with strict constraints of station and train capacities. It assumes that passengers who arrive early will have more chances to access a station and board a train. The accessing and boarding processes of passengers are all based on a first-come-first-serve basis. When a station is full, passengers unable to access must wait outside until the number of waiting passengers at platform falls below a given value. When a train is full, passengers unable to board must wait at the platform for the next train to arrive. Then, based on the simulation results, a two-stage genetic algorithm is introduced to find the best timetable. Finally, a numerical example is given to demonstrate the effectiveness of the proposed model and solution method.

\section{Introduction}

As the economy develops rapidly, deepening urbanization and the increasing urban population lead to a large demand for urban rail transit in many cities. In 2013, over 3.2 billion and 2.5 billion passenger rides were delivered by the Beijing and Shanghai subway systems, respectively; the average passenger rides on weekdays are more than 10 million and 7 million, respectively [1]. With such high demand, making urban rail transit more efficient has become a primary task for many cities. To improve the service quality of urban rail transit, a variety of operation strategies have been proposed, such as adjusting the speed of trains [2] and optimizing train formation [3]. Of all these strategies, timetable design has been accepted as the most straightforward and effective solution [4].

Timetable design problem (TDP) aims at determining a preoperational schedule for a set of trains and follows some train operational requirements [5]. The most popular technique to design timetable is to use mathematical programming, which is initialized by Amit and Goldfarb
[6] in 1971 for railway transportation and developed by a large number of researches (such as Carey and Crawford [7], Castillo et al. [8, 9], and Castillo et al. [10]).

Although there are some differences between urban public transportation and railway transportation, the mathematical programming method has still been introduced to create efficient timetables for urban public transportation to reduce passenger waiting time. Cury et al. [11], Furth and Wilson [12], Wang et al. [13], and Barrena et al. [14] proposed optimization models to design a timetable for a single line. With the expansion of subway networks, some researchers have attempted to set up a timetable for multiple services in a connected transit network (de Palma and Lindsey [15], Caprara et al. [16], Liebchen [17], Wong et al. [18], and Aksu and Akyol [19]). However, most of them assumed that the capacity of the trains is always sufficient to receive all passengers who want to enter the train and the capacity of the stations is large enough to receive all passengers who need to be evacuated. Moreover, the average waiting time of passengers is always simplified as half of the transportation headway. In fact, infinite capacity is unrealistic and 
the average waiting time of passengers may be longer when capacity constraints are considered.

In order to provide a more efficient timetable for passengers, vehicle capacity has been widely considered. Ceder [20] first addressed the importance of ridership information and stated that service frequency should correspond to temporal passenger demand. One important target of this research is to avoid overcrowding (in an average sense) on the vehicles. For a further study, Ceder [21] proposed a scheduling model to replace constant headway. In this model, the ridership of each vehicle is under an ideal value. These models, however, are not suitable for a public service with high frequency [22]. Koutsopoulos et al. [23] proposed a model to find optimal headway by minimizing social cost. In their model, vehicle capacity is indirectly incorporated in the inconvenience cost due to crowding on the vehicles, and the inconvenience cost will increase as the volume to capacity ratio increases. However, as this setup will only increase the inconvenience cost when the transit vehicle is more congested, it still allows the capacity to be exceeded. Different from the three studies above, the capacity constraints are strictly enforced in the optimization models formulated in Sun et al. [4], Niu and Zhou [24], Albrecht [25], Chen [26], and Hassannayebi et al. [27]. In their models, all passengers boarding a vehicle obey the first-come-first-serve (FCFS) principle and when a vehicle is full, passengers unable to board must wait for the next vehicle to arrive. Thus, the waiting time of a passenger is the sum of time waiting for the first coming service and for the next one as a result of previous boarding failure.

Although there is a comprehensive body of literature on TDP, few studies have drawn attention to the limitation of station capacity; also, the outside-station waiting time (OSWT) caused by being unable to get into a crowding station has always been neglected. In fact, waiting outside the station is a common phenomenon in overcrowded urban rail transit systems, such as subway systems in China. In order to provide a safe and efficient movement in stations, especially in an underground station with more enclosed and very limited internal space, operators will routinely restrict the number of passengers in stations. That is to say, some passengers will be required to wait outside the station when the number of passengers in stations exceeds the safe-value. For example, in Beijing, 63 urban rail stations mainly along Line 1, Line 5, Line 6, Line 13, Batong Line, and Changping Line instituted these restrictions since July 8, 2014, during a.m. peak hours. And OSWT in some stations, such as ShaHe, an intermediate station of Changping Line, is more than 20 minutes.

In addition, many studies in the area of TDP aim to minimize the waiting time of passengers [24-27]. This singlesided approach, however, results in timetables with high operational costs since timetables that offer the minimum waiting time for passengers usually require a high-frequency service even for transit lines with low demand. It should be noted that, although studies such as the work in [24] do not take operational interests into account explicitly, some constraints are considered to restrict the operational costs within reasonable values. A more realistic approach, which is also followed in this paper, is to minimize an objective

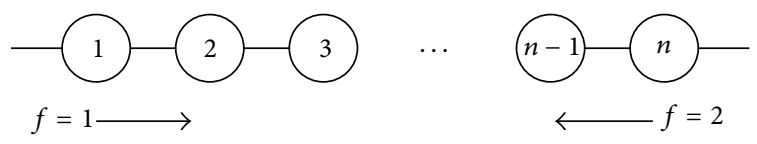

FIGURE 1: The representation of an urban transit rail line.

function that consists of both the operational and passenger waiting costs, such as the approach proposed by [19].

This paper fully considers the constraints of station and train capacities. Thus, the passenger waiting time can be divided into three parts, namely, (1) initial platform waiting time (IPWT), time spent waiting on the platform for the first coming train; (2) extra platform waiting time (EPWT), waiting time spent by left-behind passengers, who fail to board the first coming train due to the limitation of train capacity; and (3) OSWT, waiting time spent outside the station. Then, an optimization model is proposed to create an efficient and economical timetable. The contributions of this paper are summarized as follows.

(i) A simulation model is developed to simulate the movements of passengers and trains with constraints of capacities, such as train maximum capacity and station safety capacity.

(ii) The total waiting time of passengers, which includes IPWT, EPWT, and OSWT, is measured based on the outputs of the simulation model.

(iii) An optimization model is proposed to minimize the operational and passenger waiting costs; a two-stage simulation-based genetic algorithm is developed to solve the model.

The remainder of the paper is as follows. Section 2 describes the timetable design problem of urban rail line in detail. The simulation model of an urban rail line is presented in Section 3. Based on the simulation model in Section 3, a two-stage simulation-based optimization approach is proposed in Section 4. In Section 5, a numerical experiment is performed to show the application of the proposed model and solution algorithm. The final section concludes the paper and discusses future research issues.

\section{Timetable Design Problem}

2.1. Problem Description. This paper focuses on the TDP of an urban rail line with $n$ stations, as shown in Figure 1. The stations are numbered consecutively with the index values $1,2, \ldots, n$, where stations 1 and $n$ denote the start terminal and the return terminal, respectively. Each train departs from station 1 , makes a U-turn at station $n$, comes back to station 1 after a given recovery time at return terminal $n$, and then prepares for a new departure. To simplify, all trains are assumed to have the same travel time between two consecutive stations and the same dwelling time at each station. Thus, the TDP in this paper aims at determining a departure schedule for trains at the start terminal.

Let $[0, T]$ denote the study period; that is, 0 and $T$ represent the start and end of the study hours. To simplify 
the problem, the paper divides the continuous study period $[0, T]$ equally into a number of equal-length time intervals. Then, the study period $[0, T]$ can be represented as a set of discrete time points of the form $\Gamma=\{0, \delta, 2 \delta, \ldots, m \delta\}$, where $\delta$ denotes the time interval which is equal to $T / m$ and $m$ is a positive integer. To simplify the presentation, we ignore $\delta$ and simply write $\Gamma$ as $\{0,1,2, \ldots, m\}$. We also assume that all times (section running times of trains, etc.) are multiples of $\delta$. For example, a travel time of five units means, $5 \times \delta$ seconds.

Other assumptions made throughout the paper are explained as follows.

Assumption 1. The distribution of passenger demand is given and is steady during the study period.

Assumption 2. Whether a station is under an overcrowding situation is decided by the number of waiting passengers in station. That is, passengers cannot enter a station when the number of waiting passengers at station is larger than the safevalue. In fact, alighting passengers will also lead to a crowding situation at station. However, it is difficult for operators to forecast the number of alighting passengers of each train. For simplification, we only forecast the maximum possible number of alighting passengers. Then, the safe capacity for waiting passengers is equal to design capacity minus this maximum number.

Assumption 3. In general, passengers who arrive early will have more chances to access a station or board a train. In order to facilitate simplification, the paper assumes that all passengers accessing a station or boarding a train obey the FCFS principle. The passengers who fail to access an overcrowding station or fail to board a full train must wait for the next chance.

Assumption 4. The proposed model focuses on reducing passenger waiting time. The accessing walking time of passengers at a station is not considered in this model. Here, we assume that the accessing walking time of passenger is assumed to be fixed and equal to 0 .

The following notations and parameters are used throughout this paper.

Sets are as follows:

$\Gamma$ : set of time intervals,

$N$ : set of stations,

$R$ : set of trains.

Indices are as follows:

$u, v$ : index of stations,

$j$ : index of passengers,

$r$ : index of trains,

$t$ : index of modeling time intervals, $t \in \Gamma$,

$f$ : index of travel direction; let $f=1$ when a train travels from start terminal to return terminal and $f=2$ for the opposite direction.
Parameters are as follows:

$\lambda_{u}^{t}$ : number of passengers who arrive at station $u$ at time $t$

$\rho_{u v}$ : passenger destination probability, the probability of potential destination station $v$ from origin station $u$,

$q_{u}$ : total number of passengers who arrive at station $u$ during the study period,

$d_{u}$ : dwelling time at station $u$,

$s_{u, u+1}$ : running time between stations $u$ and $u+1$

$e_{1}, e_{n}$ : recovery times at start terminal and return terminal,

$r_{\max }:$ maximum number of trains

$K$ : prespecified fleet size,

$m$ : number of time intervals,

$\varphi$ : train operating cost per vehicle per unit time,

$\eta$ : waiting time cost per passenger per unit time,

$H_{\text {max }}$ : maximum service headway,

$H_{\text {min }}$ : minimum service headway,

CT: maximum capacity of trains,

$\mathrm{CS}_{u}$ : design capacity of station $u$,

$L A_{u}^{\max }$ : maximum possible number of passengers alighting at station $u$ during study period,

$\gamma_{u}$ : maximum capacity for waiting passengers at station $u, \gamma_{u}=C S_{u}-L A_{u}^{\max }$

$\theta_{u}$ : threshold which is used to judge whether passengers can access the overcrowding station $u$. Passengers waiting outside the station can access station $u$ if the number of passengers in station $u$ is less than $\theta_{u} \cdot \gamma_{u}$; otherwise, they cannot access this station,

$w_{u, t}$ : let $w_{u, t}=1$ if passenger can access the station $u$ at time $t$, and 0 otherwise.

Intermediate variables in simulation modelare as follows:

$b_{r}^{t}$ : number of boarded passengers on train $r$ at time $t$,

$L O_{u}^{t}$ : number of passengers waiting outside station $u$ at time $t$,

$L P_{u, f}^{t}$ : number of passengers waiting at time $t$ on the platform of station $u$ to board on train in direction $f$,

$L A_{u}^{t}$ : number of passengers alighting at station $u$ at time $t$, $T D_{u, f}^{r}$ : departure time of train $r$ at station $u$ in direction $f$, $T A_{u, f}^{r}$ : arrival time of train $r$ at station $u$ in direction $f$,

$T R_{t}^{u, j}$ : departure time of the first train for the direction of the $j$ th passenger who accesses the station $u$ at time $t$,

$T F_{r}$ : time by the end of the recovery operation after the train $r$ returns to the start terminal,

$P A_{u}^{j}$ : arrival time of the $j$ th passenger at station $u$,

$P C_{u}^{j}$ : accessing time of the $j$ th passenger at station $u$,

$P B_{u}^{j}$ : boarding time of the $j$ th passenger at station $u$, 
$N F_{t}$ : number of idle train unit at time $t$; initialize $N F_{0}=K$, $\sigma_{u, t}$ : let $\sigma_{u, t}=1$ if station $u$ is under an overcrowding situation at time $t$ and 0 otherwise.

Decision variable is as follows:

$x_{t}$ : let $x_{t}=1$ if a train departs from start terminal at time $t$ and 0 otherwise.

2.2. Model Formulation. The TDP in this paper aims at determining the departure time of each service at start terminal. The objective is to minimize the total cost of the transit system $C$, which is the sum of the operating cost $C^{o}$ and the passenger waiting cost $C^{w}$. Thus, the problem can then be formulated as follows:

$$
\begin{gathered}
\operatorname{Min} C=C^{o}+C^{w}, \\
C^{o}=\varphi \cdot\left(2 \cdot\left(\sum_{u=1}^{n-1} s_{u, u+1}+\sum_{u=1}^{n} d_{u}\right)+e_{1}+e_{n}\right) \cdot \sum_{t \in \Gamma} x_{t}, \\
C^{w}=\eta \cdot \sum_{u \in N} \sum_{j \in\left[1, q_{u}\right]}\left(\left(T R_{P C_{u}^{j}}^{u, j}-P C_{u}^{j}\right)+\alpha \cdot\left(P B_{u}^{j}-T R_{P C_{u}^{j}}^{u, j}\right)\right. \\
\left.+\beta \cdot\left(P C_{u}^{j}-P A_{u}^{j}\right)\right),
\end{gathered}
$$

subject to

$$
\begin{aligned}
& \sum_{t \in\left[t_{1}, t_{2}\right]} x_{t} \leq 1, \quad t_{2}=t_{1}+\frac{H_{\min }}{\delta}-1, \forall t_{1}, t_{2} \in \Gamma \\
& \sum_{t \in\left[t_{1}, t_{2}\right]} x_{t} \geq 1, \quad t_{2}=t_{1}+\frac{H_{\max }}{\delta}-1, \forall t_{1}, t_{2} \in \Gamma, \\
& x_{m}=1 \text {, } \\
& b_{r}^{t} \leq \mathrm{CT}, \quad \forall t \in \Gamma, \forall r \in R, \\
& \sum_{f=\{1,2\}} L P_{u, f}^{t}+L A_{u}^{t} \leq \mathrm{CS}_{u}, \quad \forall t \in \Gamma, \forall u \in N, \\
& w_{u, t}=\left\{\begin{array}{ll}
1 & \text { if } \sigma_{u, t}=0 \text { or } \sum_{f=\{1,2\}} L P_{u, f}^{t}<\theta_{u} \cdot \gamma_{u} \\
0 & \text { else, }
\end{array},\right. \\
& N F_{t} \geq 0, \quad \forall t \in \Gamma, \\
& L P_{u, f}^{t}=0, \quad t=T D_{u, f}^{r_{\max }}, \forall u \in N .
\end{aligned}
$$

In this formulation, the objective function (1) minimizes the total cost of transit system. Equation (2) calculates the total operation $\operatorname{cost} C^{o}$, which is the sum of the operating cost of all departure trains during the study period. Equation (3) calculates the total waiting cost of passengers $C^{w}$, which is the product of the number of passengers and their waiting time. In general, total waiting time of a passenger can be simply calculated by $P B_{u}^{j}-P A_{u}^{j}$. However, passengers may have different feelings for different kinds of waiting time [28], especially for OSWT, since passengers may need to brave the scorching sun or biting wind. Therefore, EPWT and OSWT may be presented at a higher rank. Here, two magnification factors $\alpha$ and $\beta(\alpha, \beta \geqslant 1)$ are introduced to describe this higher rank.

Constraint (4) ensures that the headway between two successive trains should meet the safety requirement. Constraint (5) ensures that the headway between two successive trains should not be too long or else passenger will have to wait long time. Constraint (6) predefines the last service of the urban rail line. Constraints (7) and (8) correspond to the limited capacity, ensuring that occupancy of trains and stations is not more than capacity. Note that it is difficult for operators to forecast the number of alighting passengers of each train. Thus, a simplified constraint, $\sum_{f=\{1,2\}} L P_{u, f}^{t}+$ $L A_{u}^{\max }<C S_{u}$, is proposed to replace the original constraint (8) based on Assumption 2. Constraint (9) guarantees that the accessing process occurs only when the station is under an undersaturated situation $\left(\sigma_{u, t}=0\right)$ or the number of passengers at the station is less than threshold $\left(\theta_{u} \cdot \gamma_{u}\right)$. Constraint (10) guarantees that there exist available train units to depart from the start terminal at scheduled departure times. Constraint (11) ensures that the train supply could meet the total passenger demands during the study period.

For our case, the movement of passengers is restricted by a series of constraints, and it is difficult to use mathematical modeling approach to describe the detailed movement of passengers or calculate passenger waiting time. As mentioned in [13], minimizing passenger waiting time is a nonlinear nonconvex objective function and it is computationally expensive to evaluate. Here, due to the nonlinear nature of the optimization problem, an alternative approach, simulation modeling, is proposed to evaluate solutions.

\section{Simulation Model}

In this section, a simulation model of urban rail line is presented to evaluate the performance of timetables. It is characterized by a discrete-event and synchronous simulation to model dynamic processes. The simulation process is illustrated in Figure 2.

As shown in Figure 2, six main events are defined in the simulation model. They can be divided into two kinds, namely, passenger dynamic event and train operation dynamic event. The details of the events are described as follows.

(1) Events concern the passenger dynamics: four main events are defined to describe passenger dynamics. The flowcharts of system events and the changes in variables related to passenger arrival, accessing, boarding, and alighting are shown in Figures 3, 4, 5, and 6, respectively. Every time a new passenger arrives at the station, he or she accesses the station based on the FCFS rule. If the number of passengers in station is more than the safe-value, the passengers are required to stay outside the station and wait for permission to access the station (Figure 4). In station, passengers stay in a queue at the platform to board incoming trains. 


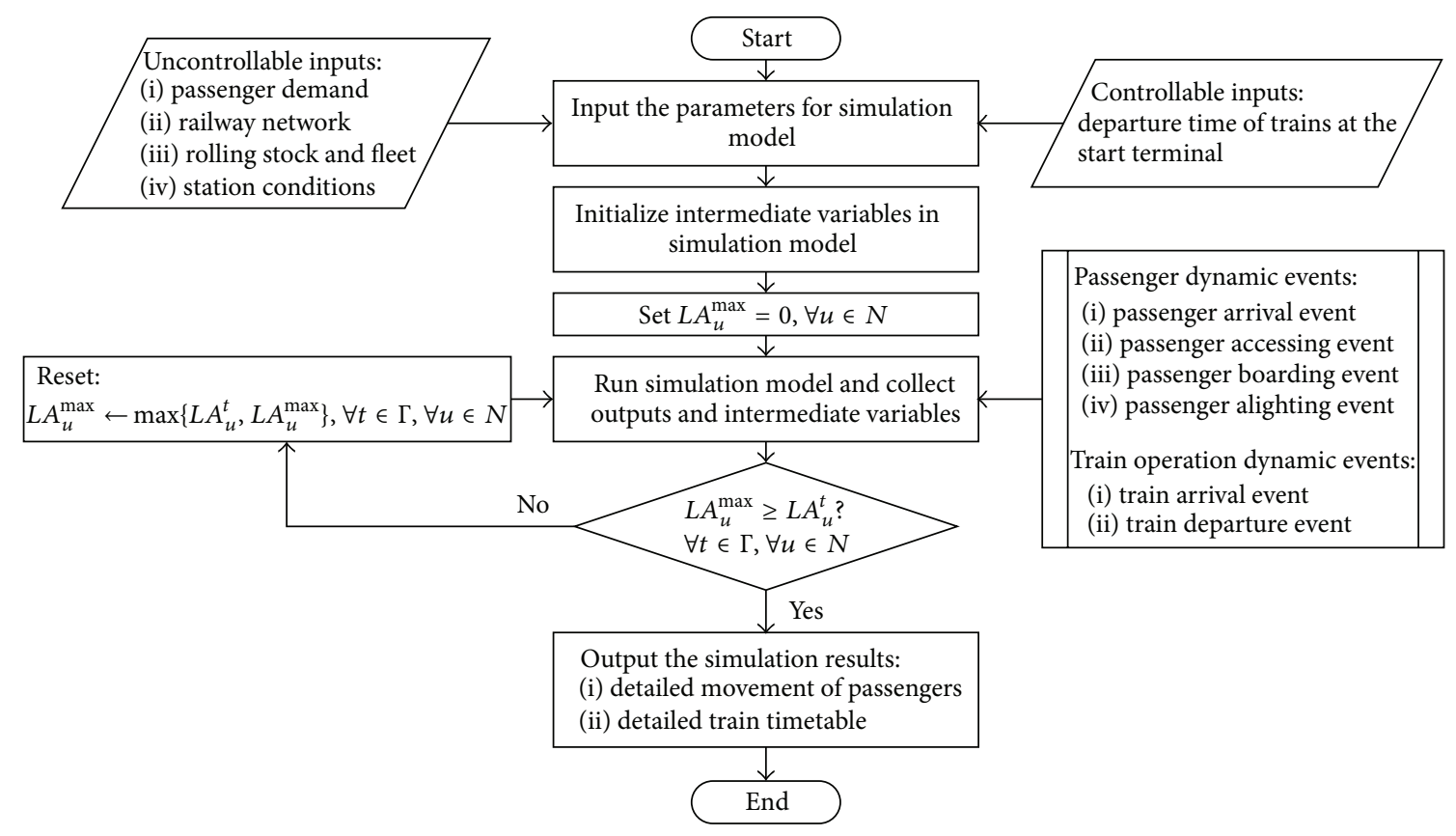

FIGURE 2: Framework of simulation model.

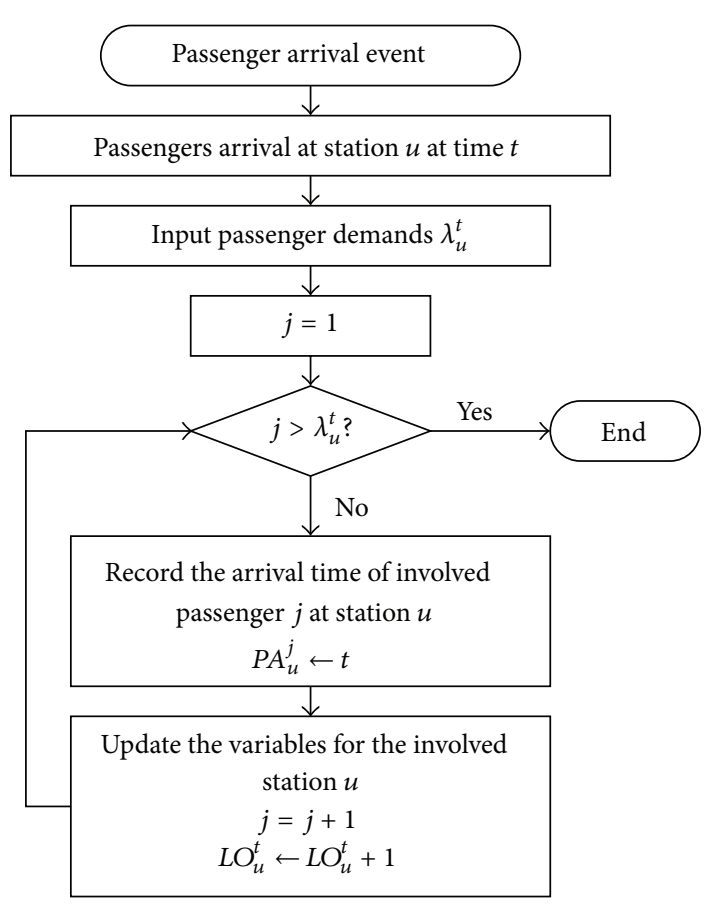

FIGURE 3: Flowchart of passenger arrival event.

The boarding processes of passengers also obey the FCFS rule. The passengers who cannot board a full train should wait for the next incoming train at the platform (Figure 5). The variables in order to handle the logics and constraints, such as the length of queues $\left(L P_{u, f}^{t}\right.$ and $\left.L O_{u}^{t}\right)$ and the load of trains $\left(b_{r}^{t}\right)$, are updated at the time events. The arrival time $\left(P A_{u}^{j}\right)$, accessing time $\left(P C_{u}^{j}\right)$, and boarding time $\left(P B_{u}^{j}\right)$ are recorded for calculating the waiting time of passengers.

(2) Events concern the train dynamics: two main events are defined to describe passenger dynamics, namely, train arrival event (Figure 7) and departure event (Figure 8). Every time when a train needs to depart from the start terminal, the number of idle train units $\left(N F_{t}\right)$ should be checked. If there exist available train units, the train can depart punctually and the number of idle train units decreases $\left(N F_{t} \leftarrow\right.$ $N F_{t}-1$ ); otherwise, the train cannot depart according to the given schedule, which means the given timetable is infeasible (Figure 8). After the train has departed from the start terminal, the details of train timetable including arrival time and departure time at each station are recorded at time events. When the train returns to the start terminal, the number of idle train units increases $\left(N F_{t} \leftarrow N F_{t}+1\right)$ after a recovery time.

Note that the maximum number of alighting passengers $\left(L A_{u}^{\max }\right)$ is an important factor, which determines the maximum capacity for waiting passengers $\left(\gamma_{u}\right)$. However, it is hard to forecast before the simulation. Thus, a feedback is designed to adjust the value of $L A_{u}^{\max }$ to ensure that the number of passengers in station is always under its design capacity.

\section{Simulation-Based Optimization Approach}

The TDP in this paper belongs to the NP-hard class (see [29]), which is difficult to solve by gradient-based methods or commercial optimization solvers. Therefore, an artificial intelligence algorithm is needed to solve the model to ensure that an optimal solution can be obtained within a reasonable amount of time.

The genetic algorithm (GA) is a stochastic search method that is inspired by the natural evolution of species. Due to its 


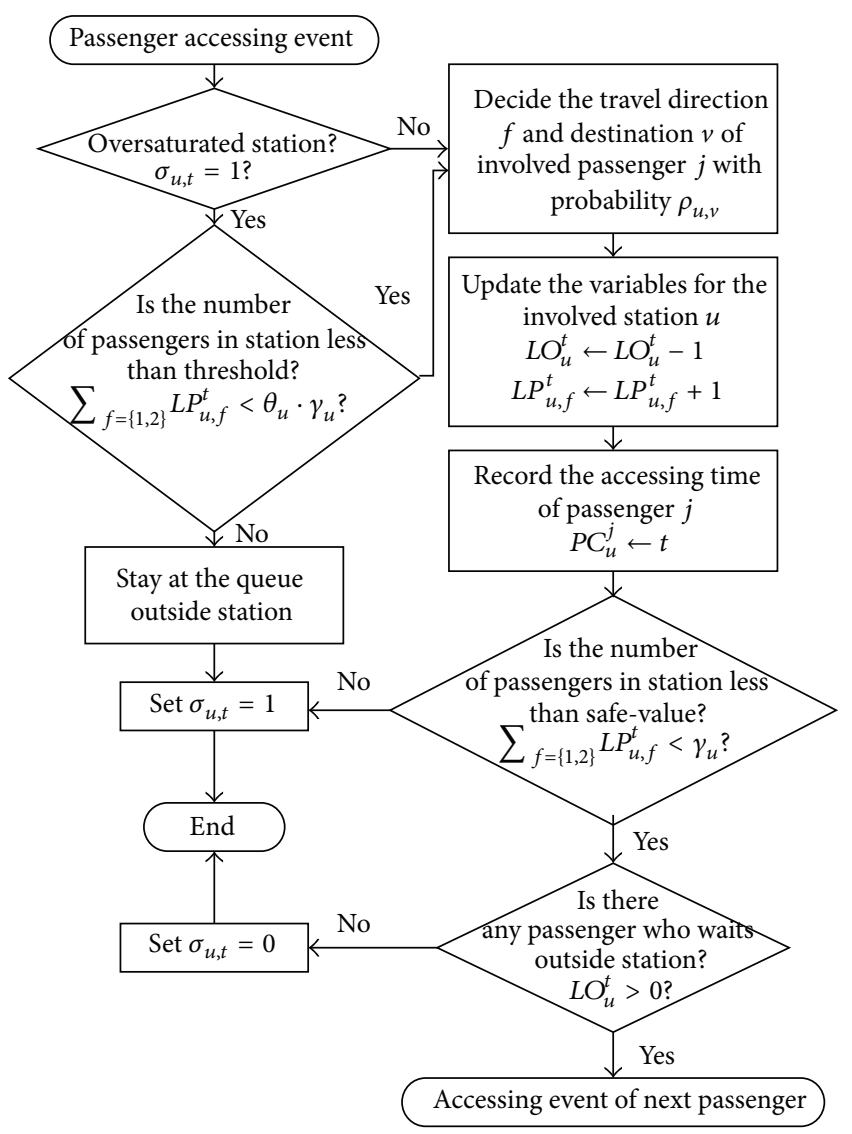

FIGURE 4: Flowchart of passenger accessing event.

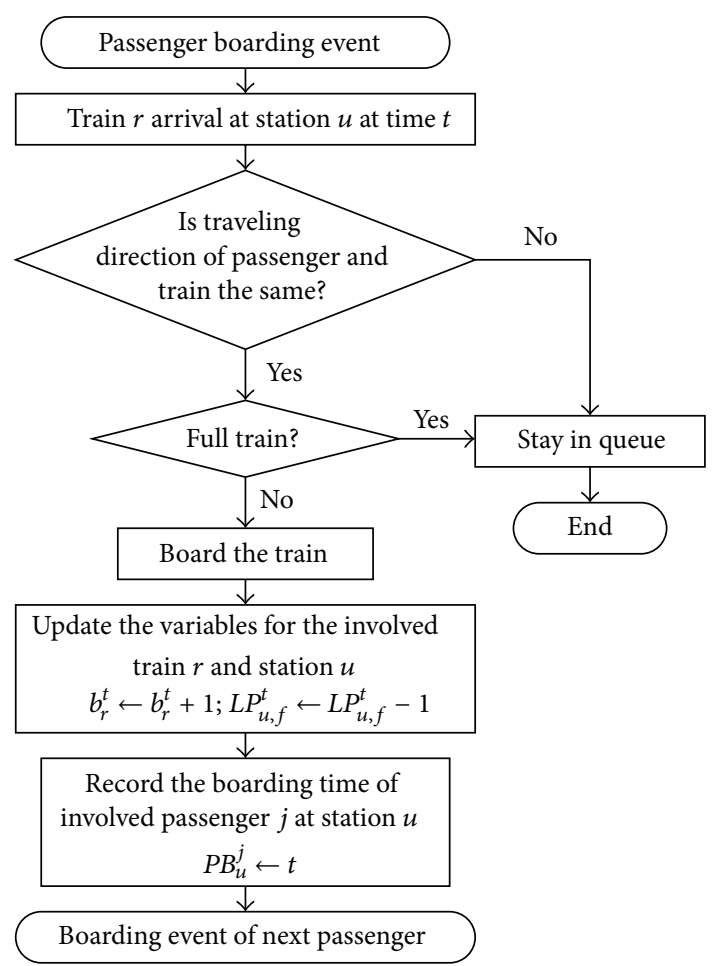

FIGURE 5: Flowchart of passenger boarding event.

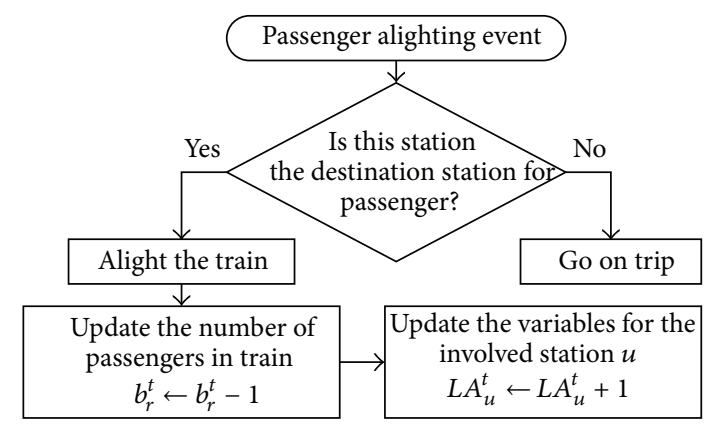

FIgURE 6: Flowchart of passenger alighting event.

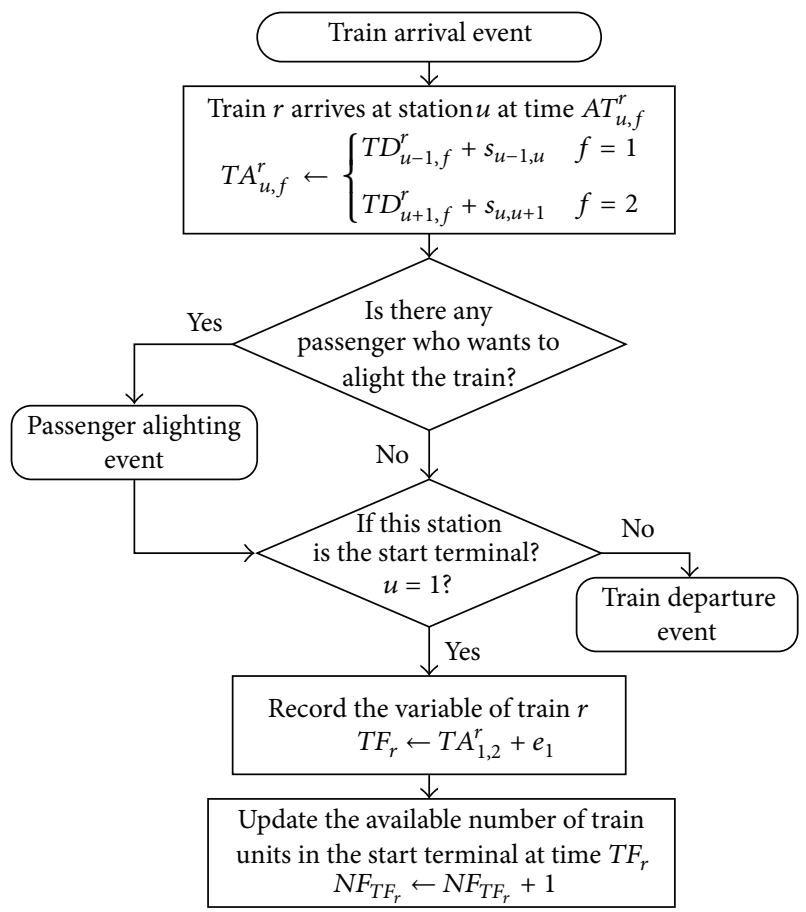

FIGURE 7: Flowchart of train arrival event.

extensive generality, strong robustness, high efficiency, and practical applicability, GA has become increasingly popular in solving complex optimization problems since the seminal work of Holland [30]. In particular, it has been successfully applied to the research on transportation systems $[5,12,14$, $19,20,22]$. In this paper, we apply GA to solve the proposed model.

4.1. Solution Representation. As the decision variable $x_{t}$ is a binary variable, a solution $X=\left(x_{1}, x_{2}, \ldots, x_{m}\right)$ can be used as a chromosome in GA directly. It is obvious that the proposed chromosome structure can represent every possible solution, and the number of departing trains changes from 0 to $m$. However, due to the constraints in the optimization model, most of the solutions are infeasible. For example, a solution with a gap or closeness between departures may not satisfy constraint (4) or (5). Thus, it is time-consuming to initialize a certain amount of feasible chromosomes randomly. In this paper, a preprocessing stage is proposed to 


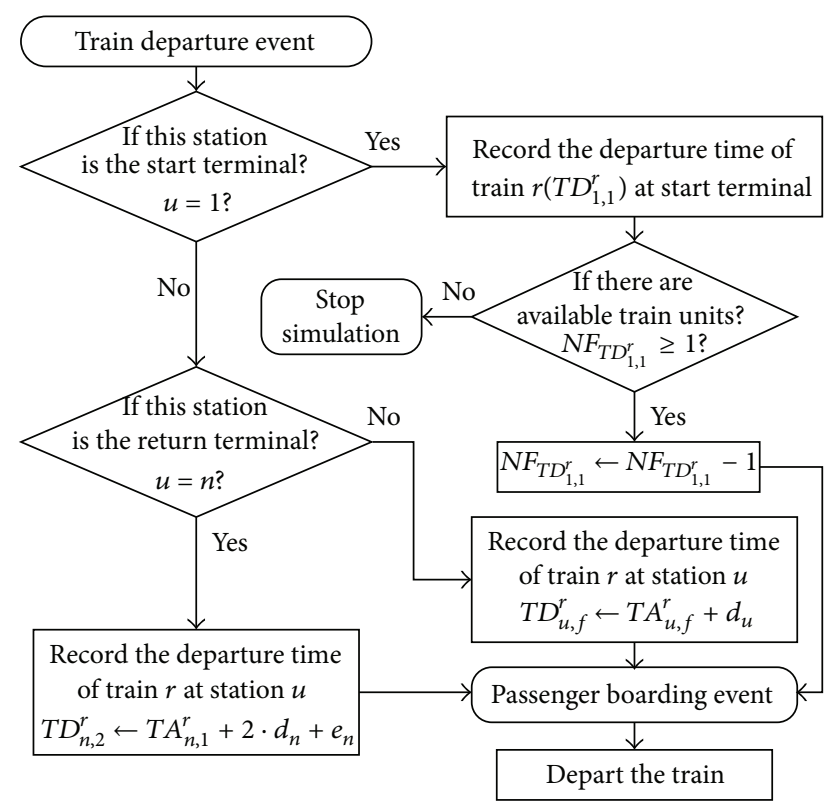

FIGURE 8: Flowchart of train departure event.

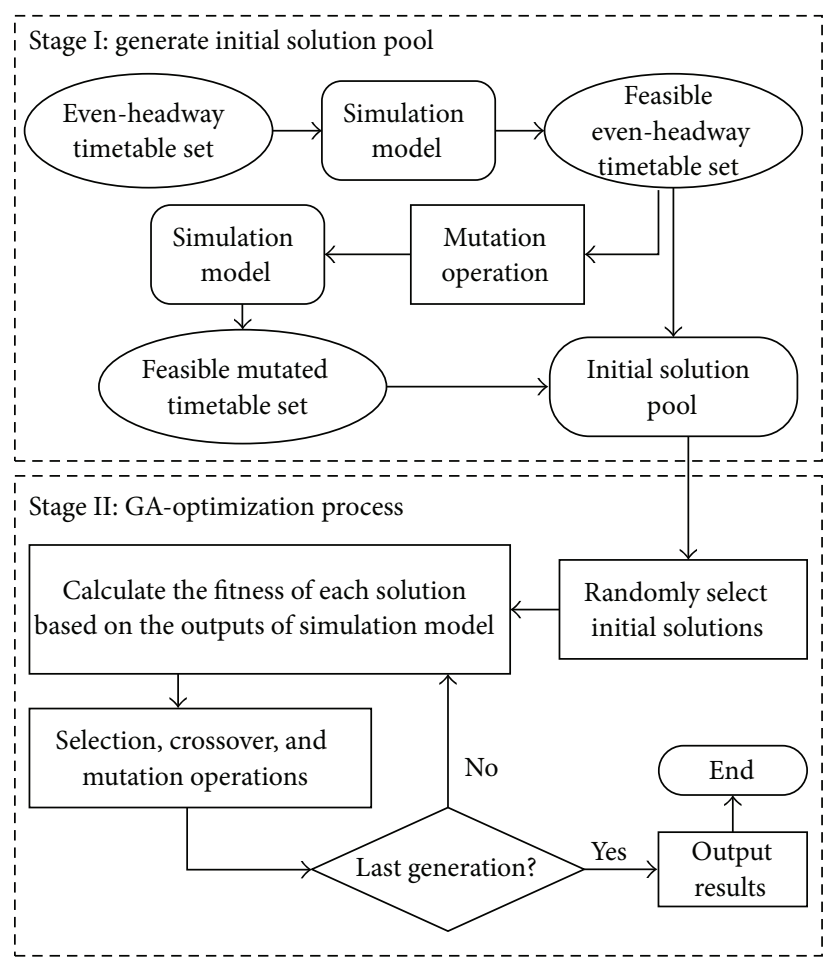

FIGURE 9: Optimization procedure integrating simulation and GA.

reduce the time spent to generate initial chromosomes. The detailed procedure of the two-stage simulation-based GAoptimization framework is demonstrated in Figure 9.

4.2. The First Stage: Generate Initial Solution Pool. An initial solution pool with pool-size chromosomes is generated in this stage. It is conceivable that even-headway timetables are

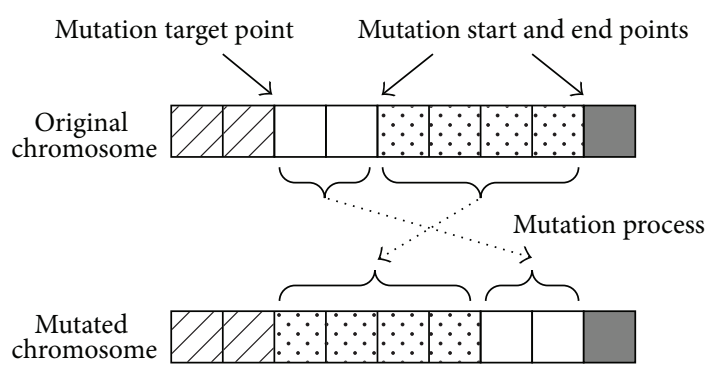

Figure 10: Mutation operation I.

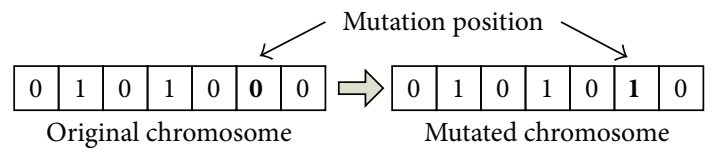

FIGURE 11: Mutation operation II.

solutions for the optimization model. Thus, the scheduled services that run with an even-headway are in question first, and the simulation experiments will be conducted to determine the feasible even-headway timetables. In this paper, a timetable is feasible if it satisfies all constraints (4)(11). Then, add feasible even-timetables to the initial solution pool. Finally, a mutation operation is introduced to enrich the initial solution pool.

Mutation Operation. In order to guarantee the diversity and availability of the mutated timetable, two mutation principles are proposed as shown in Figures 10 and 11, respectively. The algorithm of mutation is described as follows. (1) Randomly select a chromosome from feasible even-headway timetable set as the parent for mutation. (2) Randomly generate a real number $a \in[0,1]$. If $a<0.5$, obtain a mutated chromosome based on mutation operator I; otherwise, obtain a mutated chromosome based on mutation operator II.

4.3. The Second Stage: GA-Optimization Process. The procedure for GA includes the following. (1) Initialize a population with pop-size chromosomes, which are selected from the initial solution pool randomly. (2) Calculate evaluation function value $\operatorname{Eval}\left(X_{i}\right)$ of chromosome $i$ in the population based on the output of the simulation model. Here, we use objective function (1) as the evaluation function. The best individual is the chromosome with the minimum value of $\operatorname{Eval}(X)$. (3) Obtain fine chromosomes via selection, crossover, and mutation operations. (4) Terminate when the maximum number of generations is reached. The flowchart of GA is demonstrated in Figure 9.

Mutation Operation. Different from the mutation operation in the first stage, mutation operation in this stage occurs with a certain probability $P_{m}$. Randomly select a chromosome and generate a real number $a \in[0,1]$ randomly. If $a<$ $P_{m}$, a mutated chromosome is obtained based on mutation operator I or mutation operator II. And then take it to replace 


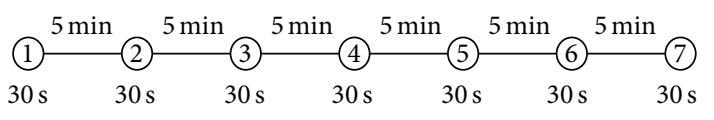

Figure 12: An urban rail line with 7 stations.

the original one if it is feasible; otherwise, keep the original one.

Other detailed steps or approaches of GA, such as selection and crossover processes, are similar to the standard GA, and interested readers are referred to the related references (e.g., Gen and Cheng [31]).

\section{Numerical Example}

5.1. Input of Date and Parameter Settings. In order to show applications of the proposed model and solution algorithm, an urban rail line with seven stations, which is shown in Figure 12, is adopted. The stations are numbered consecutively with notations $1,2,3,4,5,6$, and 7 , where stations 1 and 7 denote the start terminal and the return terminal, respectively. All the stations have an island platform. The section running time between two successive stations and dwelling time of each station are fixed at $5 \mathrm{~min}$ and $30 \mathrm{~s}$, respectively. The recovery time at the start terminal and the return terminal is taken as $90 \mathrm{~s}$; that is, $e_{1}=e_{7}=90 \mathrm{~s}$. The design capacity $\left(\mathrm{CS}_{u}\right)$ and control threshold $\left(\theta_{u}\right)$ of the station $u(u \in N)$ are taken as 1800 and 0.7.

In this example, we aim to determine the departure time of each service at station 1 during the morning peak period [7:00, 8:30]. For the convenience of the expression of passenger demand, we use "second" as a basic unit to describe the study period. That is, 0 denotes the time 7:00, 3600 denotes the time 8:00, and the representations of other times can be deduced by analogy. Then, after determining the length of modeling time interval $\delta$, the passenger demand of station $u$ at the $t$ th time interval (i.e., time period $(\delta(t-1), \delta t]$ ) can be calculated by

$$
\lambda_{u}^{t}=\psi_{u} \cdot \int_{\delta(t-1)}^{\delta t} \frac{1}{\sqrt{2 \pi} \sigma_{u}} \cdot \exp \left(-\frac{\left(\tau-\mu_{u}\right)^{2}}{2 \sigma_{u}^{2}}\right) \mathrm{d} \tau
$$

where $\psi_{u}, \mu_{u}$, and $\sigma_{u}$ are characteristic parameters of station $u$, and the values of them are shown in Table 1 . Here, we give an example of how to calculate. If we let $\delta=180 \mathrm{~s}$, the passenger demand of station 1 at the first time interval (i.e., time period $(0,180])$ will be 443 passengers, which can be obtained by the formula above with the value of $\psi_{u}, \mu_{u}$, and $\sigma_{u}\left(\psi_{u}=19800, \mu_{u}=1680\right.$, and $\left.\sigma_{u}=2700\right)$ in Table 1 .

Another important parameter related to demand, passenger destination probability $\rho_{u v}$, is depicted in Table 2 . As we can see, $10 \%$ of the passengers enter the urban rail transit system from station 3 and then travel to station 1 .

Other necessary parameters used in the simulation model and GA are summarized in Table 3. $P_{c}$ and $P_{m}$ denote the crossover and mutation rate, respectively.

All experiments in this paper are tested on a personal computer with an Inter Celeron G1620 2.7 GHz and $2 \mathrm{~GB}$
TABLE 1: Value of demand related parameters.

\begin{tabular}{cccccccc}
\hline & \multicolumn{7}{c}{$u$} \\
& 1 & 2 & 3 & 4 & 5 & 6 & 7 \\
\hline$\psi_{u}$ & 19800 & 18000 & 12600 & 3000 & 18000 & 15600 & 10200 \\
$\mu_{u}$ & 1680 & 1680 & 1800 & 900 & 1800 & 2100 & 1800 \\
$\sigma_{u}$ & 2700 & 2700 & 2100 & 4200 & 3300 & 3600 & 3600 \\
\hline
\end{tabular}

TABLE 2: Passenger destination probability $\rho_{u v}$.

\begin{tabular}{|c|c|c|c|c|c|c|c|c|}
\hline \multirow{2}{*}{\multicolumn{2}{|c|}{ From }} & \multicolumn{7}{|c|}{ To } \\
\hline & & \multirow[b]{2}{*}{1} & \multirow[b]{2}{*}{2} & \multirow[b]{2}{*}{3} & \multicolumn{2}{|l|}{$v$} & \multirow[b]{2}{*}{6} & \multirow[b]{2}{*}{7} \\
\hline & & & & & 4 & 5 & & \\
\hline \multirow{7}{*}{$u$} & 1 & - & 0.05 & 0.05 & 0.05 & 0.2 & 0.4 & 0.25 \\
\hline & 2 & 0.05 & - & 0.05 & 0.1 & 0.2 & 0.3 & 0.3 \\
\hline & 3 & 0.1 & 0.05 & - & 0.05 & 0.1 & 0.35 & 0.35 \\
\hline & 4 & 0.05 & 0.1 & 0.05 & - & 0.25 & 0.2 & 0.35 \\
\hline & 5 & 0.1 & 0.1 & 0.05 & 0.05 & - & 0.3 & 0.4 \\
\hline & 6 & 0.05 & 0.05 & 0.05 & 0.05 & 0.4 & - & 0.4 \\
\hline & 7 & 0.2 & 0.2 & 0.05 & 0.15 & 0.2 & 0.2 & - \\
\hline
\end{tabular}

RAM. The simulation-based optimization model is coded in MATLAB 7.11.

5.2. Optimization Results with Different Time Intervals. In this section, we solve the optimization problem with different time intervals, namely, $\delta=5 \mathrm{~s}, 10 \mathrm{~s}$, and $30 \mathrm{~s}$. The optimization process of GA is shown in Figure 13, and the comparison between different optimal results is shown in Table 4. Several conclusions are put forward here.

(1) The objective value is obtained using numerical calculation of the operation cost and passenger waiting cost in the system. By optimizing the objective, the scheduled timetable makes a balance between operators and passengers. Figure 13 shows that the proposed two-stage GA in this paper is convergent and the optimal objective can be obtained before 70 generations.

(2) The objective value $C$ and the CPU time are different with different time intervals. And the shorter the time interval $\delta$ is, the better the objective value can be obtained and the longer the CPU time will be needed. As shown in Table 4, the best found value 15503.92 USD is obtained with the minimum time interval $(\delta=5 \mathrm{~s}$ ) with the longest CPU time $5834.57 \mathrm{~s}$. Table 5 gives the departure time of trains at the start terminal with the best found value.

(3) In order to show the superiority of the model, the optimal results are also compared with the best even-headway timetable (shown in Table 4). The results show that the proposed optimization model can not only reduce passenger waiting cost but also reduce operational cost, and the total cost of the transportation system has been reduced by almost $15 \%$, which clearly demonstrates the effectiveness of the proposed optimization model. The main reason of this result is that, comparing with even-headway timetable, the proposed optimization model is more flexible that it can adjust the departure time of each train, and the headways between any two consecutive departures vary more appropriately based 
TABLE 3: Parameters used in numerical example.

\begin{tabular}{lccccccccccccc}
\hline Parameters & $K$ & $H_{\max }$ & $H_{\min }$ & $\mathrm{CT}$ & $\varphi$ & $\eta$ & $\alpha$ & $\beta$ & Pop-size & Pool-size & Max-generation & $P_{c}$ & $P_{m}$ \\
\hline Value & 40 & $15 \mathrm{~min}$ & $120 \mathrm{~s}$ & 1680 & $640 \mathrm{USD} / \mathrm{h}$ & $1 \mathrm{USD} / \mathrm{h}$ & 1 & 1 & 40 & 260 & Up to 70 & 0.9 & 0.2 \\
\hline
\end{tabular}

TABLE 4: Comparison between different optimal results.

\begin{tabular}{lcccc}
\hline$\delta(\mathrm{s})$ & $C^{w}(\mathrm{USD})$ & $C^{o}(\mathrm{USD})$ & $C(\mathrm{USD})$ & $\mathrm{CPU}(\mathrm{s})$ \\
\hline$\delta=5$ & 2810.59 & 12693.33 & $15503.92(85.2 \%)$ & 5834.57 \\
$\delta=10$ & 2841.84 & 12693.33 & $15535.17(85.4 \%)$ & 2768.51 \\
$\delta=30$ & 3136.25 & 12693.33 & $15829.58(87.0 \%)$ & 929.09 \\
Even-headway & 3259.35 & 14933.33 & $18192.69(100 \%)$ & 281.36 \\
\hline
\end{tabular}

TABLE 5: Best solution: departure times of trains at the start terminal.

\begin{tabular}{lc}
\hline Train & Departure time \\
\hline 1 & $7: 04: 55$ \\
2 & $7: 09: 30$ \\
3 & $7: 14: 20$ \\
4 & $7: 18: 20$ \\
5 & $7: 21: 40$ \\
6 & $7: 25: 10$ \\
7 & $7: 29: 10$ \\
8 & $7: 33: 10$ \\
9 & $7: 37: 10$ \\
10 & $7: 41: 30$ \\
11 & $7: 47: 30$ \\
12 & $7: 51: 30$ \\
13 & $7: 56: 35$ \\
14 & $8: 02: 25$ \\
15 & $8: 08: 50$ \\
16 & $8: 16: 45$ \\
17 & $8: 30: 00$ \\
\hline
\end{tabular}

on the time-varying demand. As we can see in Table 5, the headways in the optimal timetable range from $200 \mathrm{~s}$ to $795 \mathrm{~s}$.

5.3. Analysis of the Impact of Station Capacity. Station capacity is an important constraint in our model. In order to analyze the impact of station capacity $\mathrm{CS}_{u}$, sensitivity analysis is conducted with $\delta=30 \mathrm{~s}$ against $\mathrm{CS}_{u}=1000,1100, \ldots$, 1700, 1800. The results are shown in Figure 14.

As we can see, when $\mathrm{CS}_{u} \leq 1400$ pax, a decreasing trend in system cost can be observed with the growth of station capacity. The total system cost improved from 29777.60 USD to 15837.34 USD when the capacities of stations increase from 1000 pax to 1400 pax. When $\mathrm{CS}_{u}>1400$ pax, however, the system cost remains at a relatively stable value (about 15854 USD). This is mainly because the larger the station capacity is, the fewer the passengers will need to wait outside station and the less the outside waiting time a passenger will have. Also, fewer trains used to quicken the decreasing of the number of waiting passengers at platform are needed. Just as shown in Figure 14, the operational cost decreases nearly monotonically from 17920.00 USD to 12693.33 USD (i.e., the

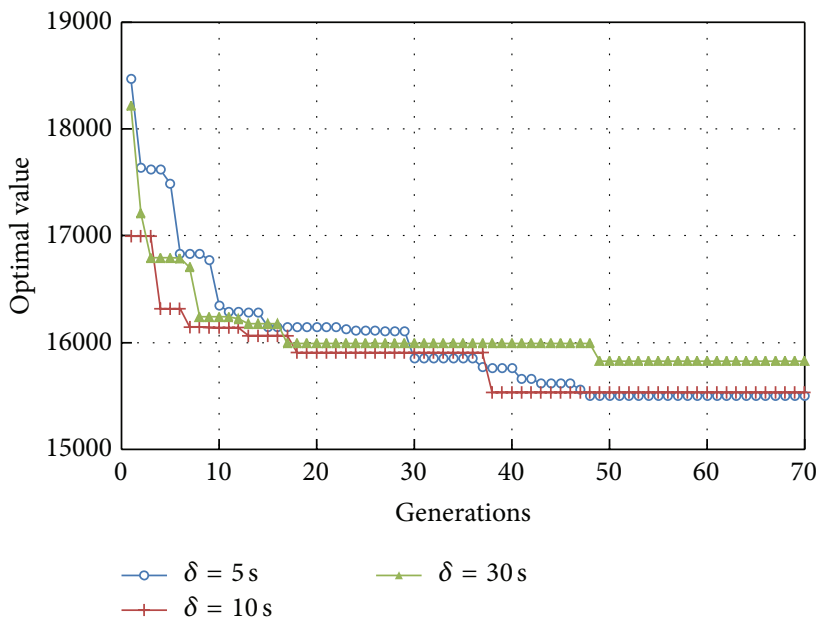

FIgURE 13: Optimization process of GA.

number of departure trains decreases from 24 to 17) with the increase of station capacity. Then, the system cost will decrease. However, when a particular value $\left(\mathrm{CS}_{u}=1400\right.$ pax in this example) is reached, the total waiting time of passenger outside station will become rather small, which is difficult to have influence on the optimal result. Thus, the system cost will not change evidently.

These results indicate that a limited station capacity will have a great influence on the service quality and operation plan of urban rail transit. And the smaller the station capacity is the higher system cost will be. However, on the other side, a too large capacity for a station will contribute little to the improving of transportation system but may bring a high infrastructure cost. Therefore, it is necessary to preestimate the system cost in the operation stage before building the station.

\section{Conclusions}

In this paper, we present a scheduling approach for a heavily congested urban rail line. It aims to create an efficient timetable with minimal passenger waiting cost and operational cost. In order to evaluate the performance of the created timetable, a simulation model is proposed with strict constraints on train and station capacities. Then, based on the simulation results, a two-stage GA is designed to find the best timetable. Finally, the feasibility of the solution method is demonstrated through a numerical example.

Although only a small case is discussed in this paper, it is shown that the strict constraint of station capacity is essential for TDP; the timetable designed by the proposed model will have a better balance between passengers and operators.

In addition, the passenger demand in our work is assumed to be steady. However, in real work, timetables will 


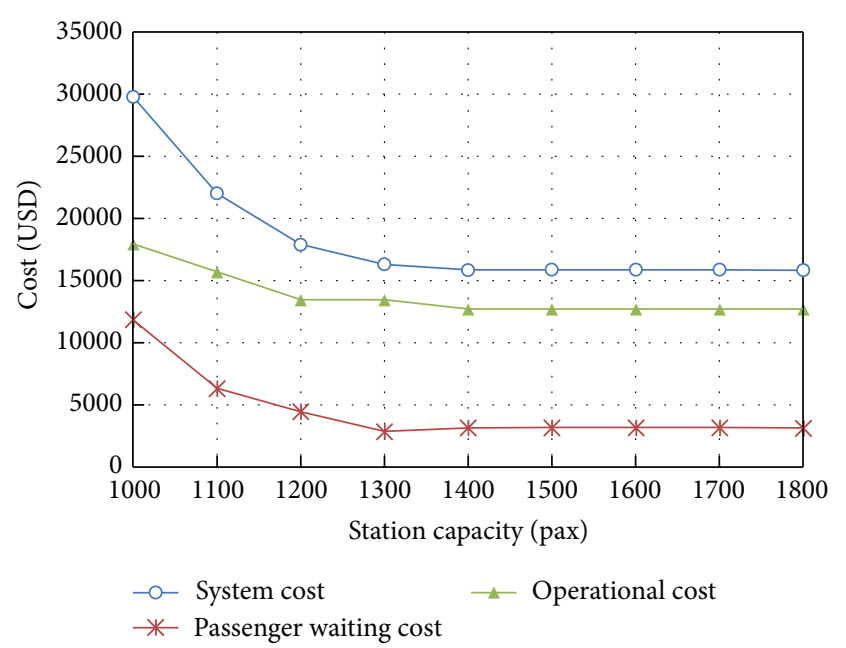

FIgURE 14: Optimal values with different station capacities.

have a feedback on the distribution of passenger demand. In our future research, we will try to create a new timetable considering this feedback.

\section{Conflict of Interests}

The authors declare that there is no conflict of interests regarding the publication of this paper.

\section{Acknowledgments}

This research was funded by the Nation Basic Research Program of China (no. 2012CB725406) and the National Natural Science Foundation of China (nos. 71131001 and 71201007). The authors also thank the anonymous reviewers and the editor for their suggestions to improve this paper.

\section{References}

[1] Research Group of Annual Report of China Urban Mass Transit, 2013 Annual Report of China Urban Mass Transit, Beijing Jiaotong University Press, Beijing, China, 2014.

[2] X. Feng, B. Mao, X. Feng, and J. Feng, "Study on the maximum operation speeds of metro trains for energy saving as well as transport efficiency improvement," Energy, vol. 36, no. 11, pp. 6577-6582, 2011.

[3] X. Feng, H. Liu, H. Zhang, B. Wang, and Q. Sun, "Rational formations of a metro train improve its efficiencies of both traction energy utilization and passenger transport," Mathematical Problems in Engineering, vol. 2013, Article ID 643274, 7 pages, 2013.

[4] L. Sun, J. G. Jin, D.-H. Lee, K. W. Axhausen, and A. Erath, "Demand-driven timetable design for metro services," Transportation Research Part C: Emerging Technologies, vol. 46, pp. 284-299, 2014.

[5] L. Kang, J. Wu, H. Sun, X. Zhu, and B. Wang, "A practical model for last train rescheduling with train delay in urban railway transit networks," Omega, vol. 50, pp. 29-42, 2015.

[6] I. Amit and D. Goldfarb, "The timetable problem for railways," Developments in Operations Research, vol. 2, pp. 379-387, 1971.
[7] M. Carey and I. Crawford, "Scheduling trains on a network of busy complex stations," Transportation Research Part B: Methodological, vol. 41, no. 2, pp. 159-178, 2007.

[8] E. Castillo, I. Gallego, J. M. Ureña, and J. M. Coronado, "Timetabling optimization of a single railway track line with sensitivity analysis," TOP, vol. 17, no. 2, pp. 256-287, 2009.

[9] E. Castillo, I. Gallego, J. M. Ureña, and J. M. Coronado, “Timetabling optimization of a mixed double- and single-tracked railway network," Applied Mathematical Modelling, vol. 35, no. 2, pp. 859-878, 2011.

[10] E. Castillo, I. Gallego, S. Sánchez-Cambronero et al., "An alternate double-single track proposal for high-speed peripheral railway lines," Computer-Aided Civil and Infrastructure Engineering, vol. 30, no. 3, pp. 181-201, 2015.

[11] J. E. Cury, F. A. C. Gomide, and M. J. Mendes, "A methodology for generation of optimal schedules for an underground railway system," IEEE Transactions on Automatic Control, vol. 25, no. 2, pp. 217-222, 1980.

[12] P. G. Furth and N. H. M. Wilson, "Setting frequencies on bus routes: theory and practice," Transportation Research Record, vol. 818, pp. 1-7, 1981.

[13] Y. Wang, B. de Schutter, T. van den Boom, B. Ning, and T. Tang, "Real-time scheduling for single lines in urban rail transit systems," in Proceedings of the IEEE International Conference on Intelligent Rail Transportation (ICIRT '13), pp. 1-6, Beijing, China, September 2013.

[14] E. Barrena, D. Canca, L. C. Coelho, and G. Laporte, "Singleline rail rapid transit timetabling under dynamic passenger demand," Transportation Research Part B: Methodological, vol. 70, pp. 134-150, 2014.

[15] A. de Palma and R. Lindsey, "Optimal timetables for public transportation," Transportation Research Part B: Methodological, vol. 35, no. 8, pp. 789-813, 2001.

[16] A. Caprara, M. Fischetti, and P. Toth, "Modeling and solving the train timetabling problem," Operations Research, vol. 50, no. 5, pp. 851-861, 2002.

[17] C. Liebchen, "The first optimized railway timetable in practice," Transportation Science, vol. 42, no. 4, pp. 420-435, 2008.

[18] R. C. W. Wong, T. W. Y. Yuen, K. W. Fung, and J. M. Y. Leung, "Optimizing timetable synchronization for rail mass transit," Transportation Science, vol. 42, no. 1, pp. 57-69, 2008.

[19] D. T. Aksu and U. Akyol, "Transit coordination using integerratio headways," IEEE Intelligent Transportation Systems Magazine, vol. 15, no. 4, pp. 1633-1642, 2014.

[20] A. Ceder, "Bus frequency determination using passenger count data," Transportation Research Part A: General, vol. 18, no. 5-6, pp. 439-453, 1984.

[21] A. Ceder, "Bus timetables with even passenger loads as opposed to even headways," Transportation Research Record, vol. 1760, no. 1, pp. 3-9, 2001.

[22] A. Ceder, Public Transit Planning and Operation: Theory, Modeling and Practice, Butterworth-Heinemann, 2007.

[23] H. N. Koutsopoulos, A. Odoni, and N. H. M. Wilson, "Determination of headways as a function of time varying characteristics on a transit network," in Computer Scheduling of Public Transport, vol. 2, pp. 391-413, 1985.

[24] H. Niu and X. Zhou, "Optimizing urban rail timetable under time-dependent demand and oversaturated conditions," Transportation Research Part C: Emerging Technologies, vol. 36, pp. 212-230, 2013. 
[25] T. Albrecht, "Automated timetable design for demand-oriented service on suburban railways," Public Transport, vol. 1, no. 1, pp. 5-20, 2009.

[26] Q. Chen, "Global optimization for bus line timetable setting problem," Discrete Dynamics in Nature and Society, vol. 2014, Article ID 636937, 9 pages, 2014.

[27] E. Hassannayebi, A. Sajedinejad, and S. Mardani, "Urban rail transit planning using a two-stage simulation-based optimization approach," Simulation Modelling Practice and Theory, vol. 49, pp. 151-166, 2014.

[28] X.-M. Chen, Q.-X. Liu, and G. Du, "Estimation of travel time values for urban public transport passengers based on SP survey," Journal of Transportation Systems Engineering and Information Technology, vol. 11, no. 4, pp. 77-84, 2011.

[29] O. J. Ibarra-Rojas and Y. A. Rios-Solis, "Synchronization of bus timetabling," Transportation Research Part B: Methodological, vol. 46, no. 5, pp. 599-614, 2012.

[30] J. H. Holland, Adaptation in Natural and Artificial Systems, University of Michigan Press, Ann Arbor, Mich, USA, 1975.

[31] M. Gen and R. W. Cheng, Genetic Algorithms and Engineering Optimization, John Wiley \& Sons, 2000. 


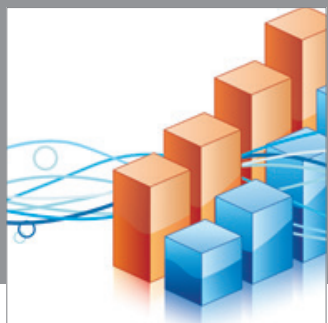

Advances in

Operations Research

mansans

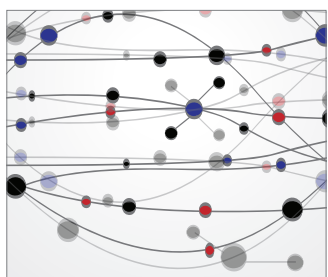

The Scientific World Journal
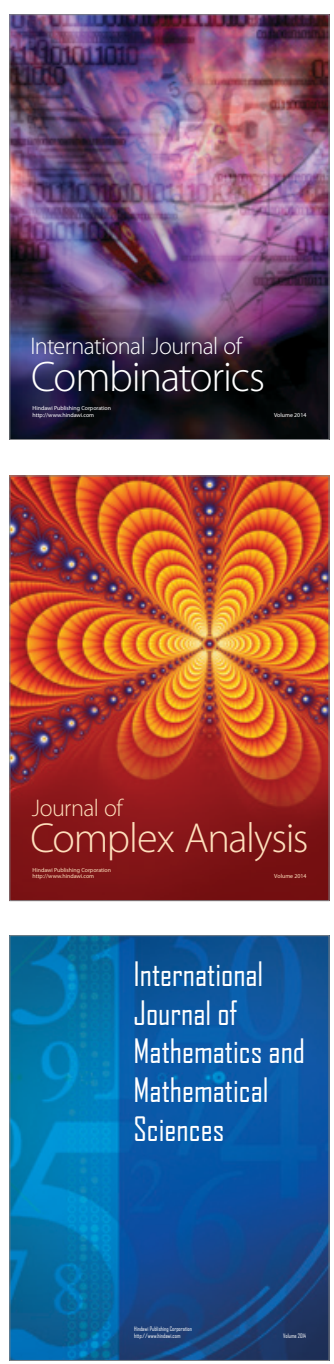
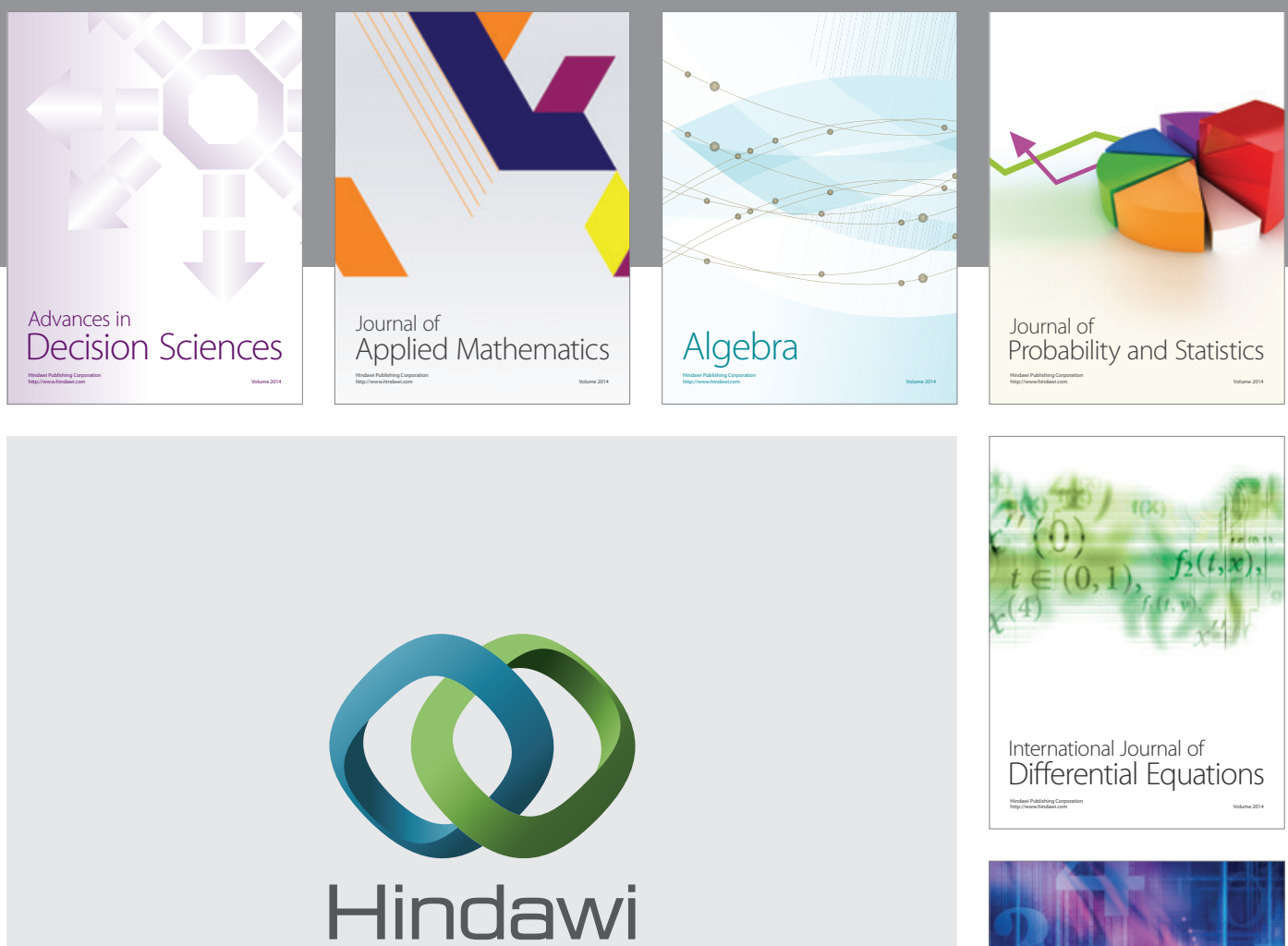

Submit your manuscripts at http://www.hindawi.com
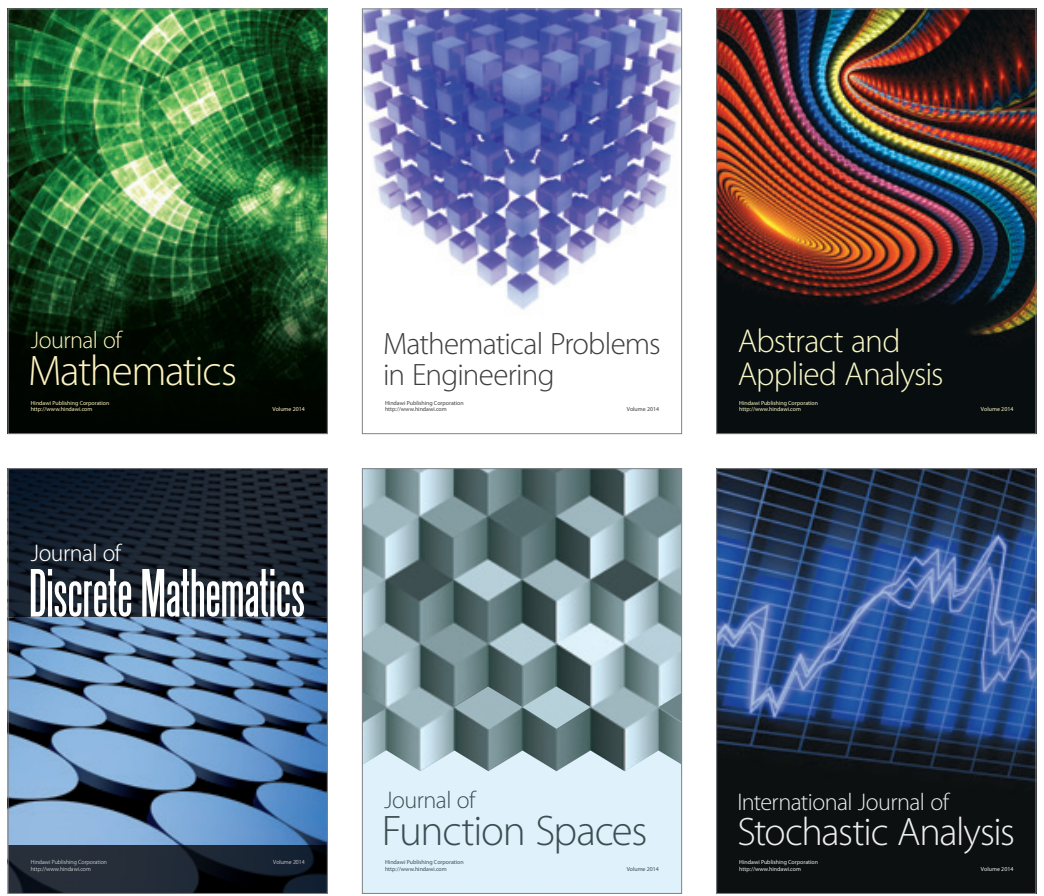

Journal of

Function Spaces

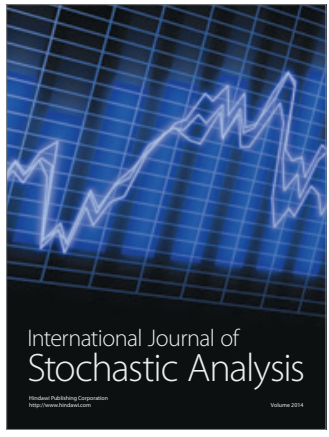

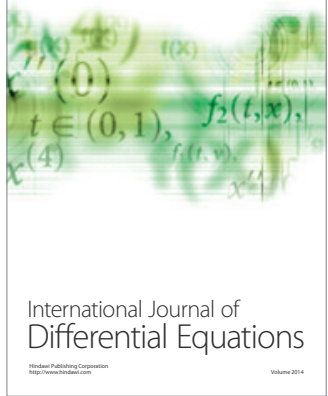
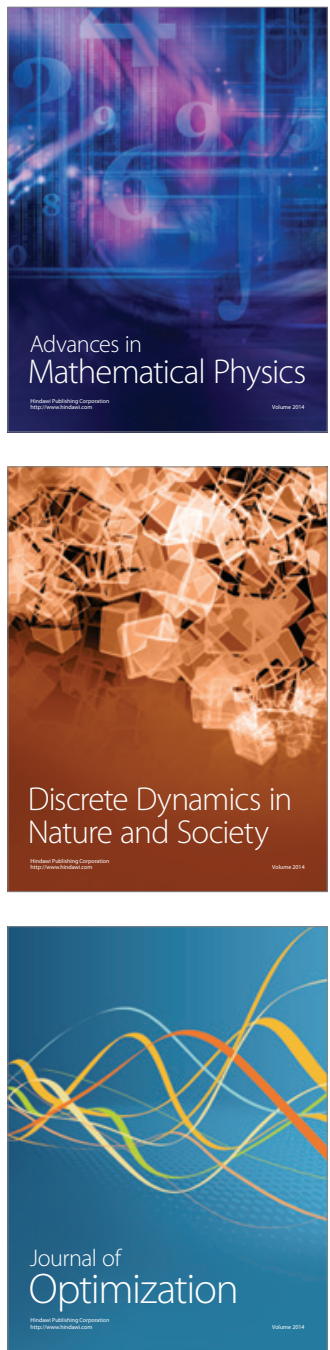